\title{
Adrenal TB: the great master of disguise!
}

\author{
Eswari Chinnasamy, Y Khalid, C Cosgrove, N Beharry \& G Bano
}

St George's University Hospitals NHS Foundation Trust

\section{Introduction}

- Delay in diagnosis of adrenal insufficiency is common. $~ 47 \%$ of patients have symptoms for over 1 year and $20 \%$ for over 5 years before diagnosis. Autoimmunity is the most common aetiology for Primary Adrenal Insufficiency (PAI) in developed countries. But up to $10-20 \%$ of PAI could be due to Tuberculosis (TB).

- TB usually affects lungs, extra pulmonary forms (EPTB) occur in up to $30 \%$ of cases. Isolated adrenal TB is relatively rare

- Adrenal glands are frequently involved in TB, adrenal insufficiency is rarely seen

\section{Case Report}

- A 42year old Afro-Caribbean male .

- 2 year history of nonspecific illness including reduced appetite, weight loss, intermittent fever presents with worsening symptoms

- After extensive inpatient investigations, he was discharged with probable viral infection and neutropaenia related to ethnicity

- Positive T-spot test. But lack of evidence of active TB at usual sites meant, he was diagnosed with latent TB, not requiring treatment

- The final diagnosis of adrenal TB was made following diagnosis of primary adrenal insufficiency and characteristic imaging findings

\section{Investigations}

FBC - Mild neutropaenia and thrombocytopaenia, occasional hyperkalaemia, normal LFTs, U\&Es and CRP. Unremarkable viral screen, haematological investigations including bone marrow aspirate for TB culture. Induced sputum AFBs and CXR were clear. Reactive T spot test. CT CAP - marked adrenal hyperplasia.

Endocrine Investigations

- $\quad 24 \mathrm{hr}$ urinary metanephrines were normal

- Low baseline cortisol on overnight

Dexamethasone suppression \& intermittent mild hyperkalaemia prompted adrenal insufficiency

- Synacthen Test:

$30 \mathrm{~min}$ Cortisol $113 \mathrm{nmol} / \mathrm{L}$

Baseline ACTH: $1213 \mathrm{ng} / \mathrm{L}$

- Aldosterone: $<89 \mathrm{pmol} / \mathrm{L}$

Renin $13 \mathrm{nmol} / \mathrm{l} / \mathrm{hr}$
Pre Treatment CT adrenal

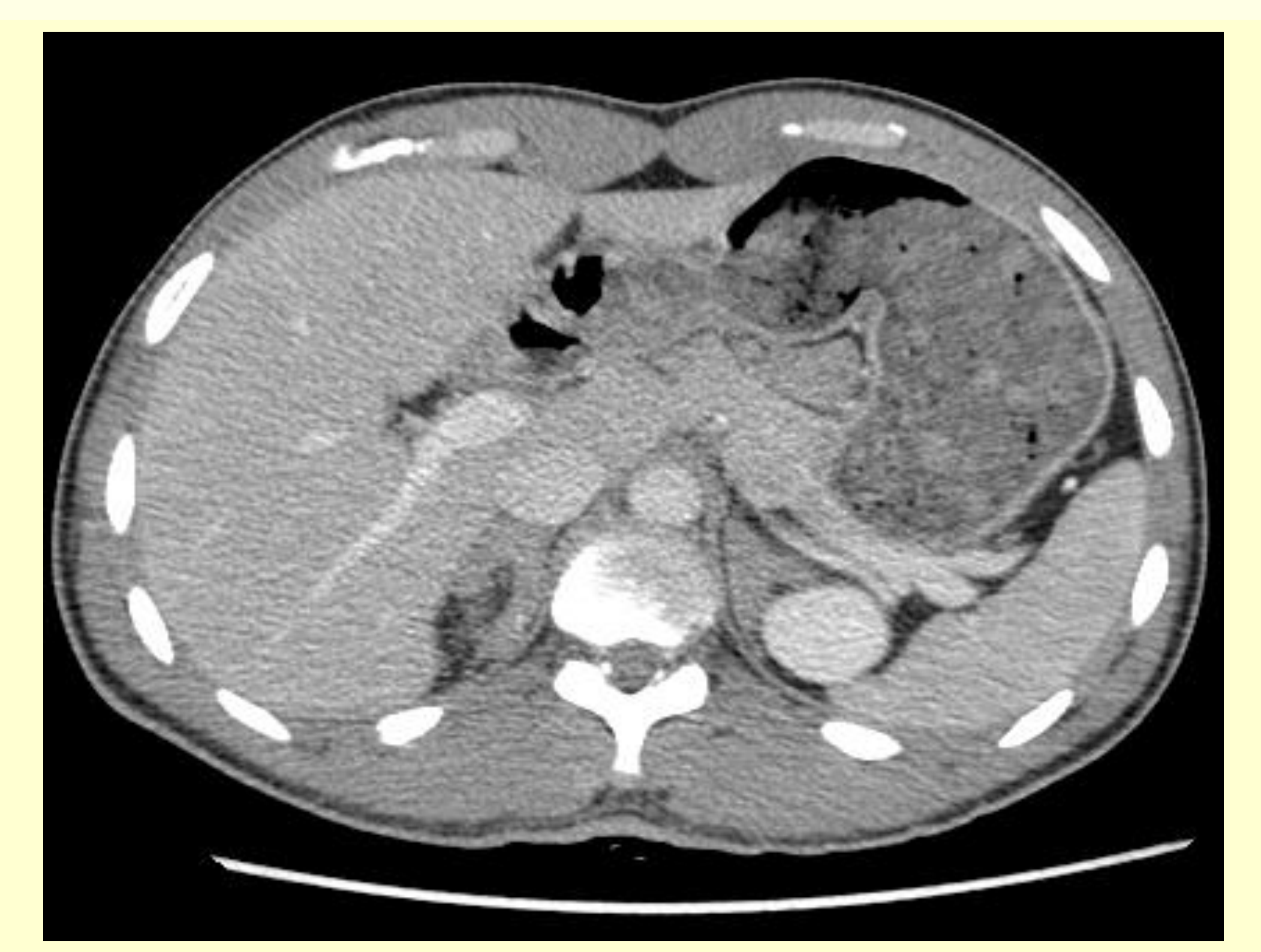

Post Treatment CT adrenal

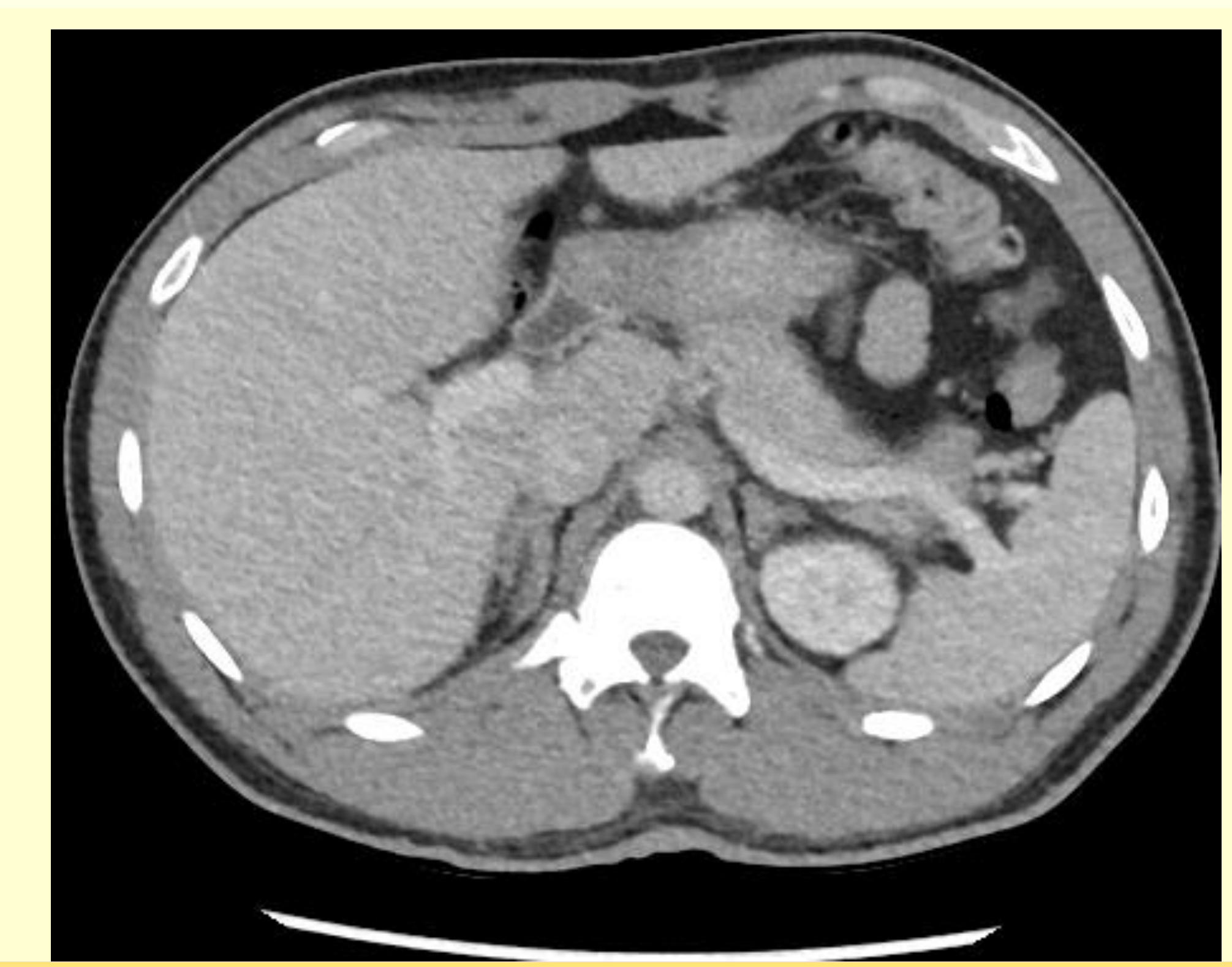

\section{Outcome}

- He felt remarkably better after commencing Hydrocortisone and Fludrocortisone

- Gradually regained his weight

- Given the imaging characteristics, isolated adrenal TB was the likely aetiology for PAI. After MDT discussion, he was commenced on 6 months Anti-tuberculous therapy.

- Hydrocortisone was doubled while on Rifampicin

- Follow up imaging show continued resolution of his adrenal hyperplasia.

- He remains on steroid replacement as his adrenal function has not recovered. This is not an uncommon outcome in TB adrenal cases

\section{Discussion}

- Thomas Addison in 1855 published 'On the Constitutional and Local Effects of Disease of the Suprarenal Capsules' while investigating a peculiar form of anaemia, majority of these cases were due to TB.

- More than $90 \%$ of the gland must be destroyed before insufficiency appears.

- Bilateral involvement is typical, initially glands are enlarged but later become atrophic with calcification

- Adrenal biopsy can be considered

- Recovery of adrenal function is very uncommon in this group

- To conclude, in anyone with PAI and bilateral adrenal enlargement \pm calcification , adrenal TB needs to be ruled out

\section{Referencès}

